\title{
Synthesis and Evaluation of 2-Amino-4H-Pyran-3-Carbonitrile Derivatives as Antitubercular Agents
}

\author{
Chunxia Chen ${ }^{1 *}$, Minghui Lu' ${ }^{2 *}$ Zhihui Liu ${ }^{3}$, Junting Wan², Zhengchao Tu², \\ Tianyu Zhang ${ }^{2 \#}$, Ming Yan ${ }^{1 \#}$ \\ ${ }^{1}$ Institute of Drug Synthesis and Pharmaceutical Process, School of Pharmaceutical Sciences, \\ Sun Yat-sen University, Guangzhou, China \\ ${ }^{2}$ State Key Laboratory of Respiratory Diseases, Guangzhou Institutes of Biomedicine and Health, \\ Chinese Academy of Science, Guangzhou, China \\ ${ }^{3}$ Guangzhou Chest Hospital, Guangzhou, China \\ Email: ${ }^{*}$ zhang_tianyu@gibh.ac.cn, ${ }^{\#}$ yanming@mail.sysu.edu.cn
}

Received March 2, 2013; revised April 2, 2013; accepted April 9, 2013

Copyright (C) 2013 Chunxia Chen et al. This is an open access article distributed under the Creative Commons Attribution License, which permits unrestricted use, distribution, and reproduction in any medium, provided the original work is properly cited.

\begin{abstract}
A series of 2-amino-4H-pyran-3-carbonitrile derivatives were designed and synthesized. Their antitubercular activities were evaluated against autoluminescent $M$. tuberculosis $\mathrm{H} 37 \mathrm{Ra}$ and standard strain M. tuberculosis $\mathrm{H} 37 \mathrm{Rv}$. No obvious antitubercular activities could be observed (MIC $>10 \mu \mathrm{g} / \mathrm{mL}$ ). The results are in sharp contrast with the previously reported data.
\end{abstract}

Keywords: 2-Amino-4H-Pyran-3-Carbonitrile; Synthesis; Antitubercular Activity

\section{Introduction}

Tuberculosis (TB) is a chronic disease caused by $M y$ cobacterium tuberculosis. It continues to be a serious threat for human health $[1,2]$. Every year about two million people die of this disease and almost eight million people get tuberculosis. The present antitubercular treatment typically requires the combination of at least two first-line drugs (rifampicin, isoniazid, ethambutol and pyrazinaimde) for an extended period (6 - 12 months). The poor compliance to the rigid implementation of therapy leads to the emergence of multi-drug-resistant (MDR) and extensively drug-resistant (XDR) strains of $\mathrm{Myco}$ bacterium tuberculosis, which have brought new challenges for clinical treatment [3-6]. In addition, the rising incidence of TB and HIV co-infection makes the treatment more difficult.

The development of antitubercular agents with new action mechanism is an urgent task $[7,8]$. In recent ten years, a number of candidates have appeared with promising activities against sensitive and resistant Mycobacte-

\footnotetext{
${ }^{*}$ These authors contribute equally to this study.

"Corresponding authors.
}

rium tuberculosis strains. In 2007, Perumal and coworkers reported 2-aminopyranopyridine-3-carbonitriles as a new type of antitubercular agents (Scheme 1) [9]. Several compounds showed excellent antitubercular activity comparable with isoniazid. Recently Perumal, Sriram and co-workers also found that 1,2,4-oxadiazoles derived from 2-aminopyranopyridine-3-carbonitriles showed enhanced antitubercular activity (Scheme 1) [10]. These results strongly suggest that 2-amino-4H-pyran-3-carbonitrile is a new pharmacophore of antitubercular agents.

Recently we have developed efficient methods for the synthesis of homochiral 2-amino-4 $\mathrm{H}$-pyran-3-carbonitriles $[11,12]$. We are interested in the effect of chiral center of these compounds on the antitubercular activity. We are also interested in the further improvement of the antitubercular activity of 2-amino- $4 \mathrm{H}$-pyran-3-carbonitriles by structural modifications. In this paper, we report the synthesis of racemic and homochiral 2-amino-pyranopyridine-3-carbonitriles as well as their structural analogs. The antitubercular activity was evaluated in vitro against autoluminescent Mycobacterium tuberculosis H37Ra and standard strain Mycobacterium tuberculosis H37Rv. 

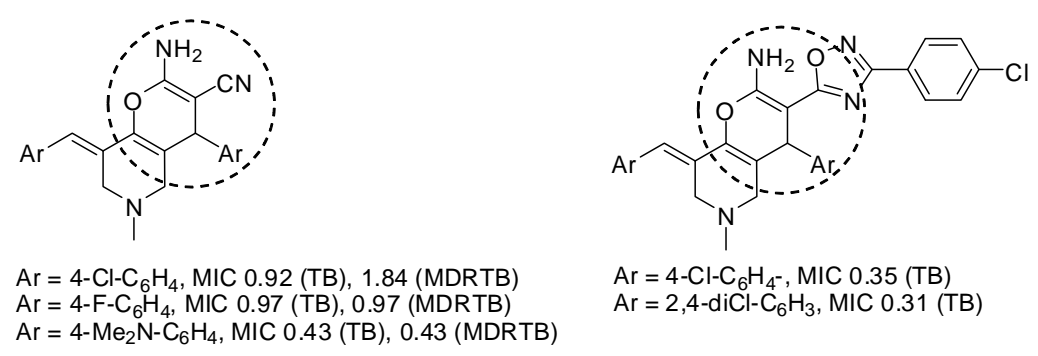

Scheme 1. 2-Amino-4H-pyran-3-carbonitrile and their derivatives with potent antitubercular activity.

\section{Results and Discussion}

\subsection{Chemistry}

Racemic 2-aminopyranopyridine-3-carbonitriles 2a-2g were prepared from dienones 1a-1g and malononitrile in the presence of piperidine. Generally excellent yields $(92 \%$ 99\%) were obtained (Scheme 2).

To explore the effect of chiral centers in $\mathbf{2 a - 2 g}$, homochiral $(S)$-2a, $(S)$-2d, $(R)-\mathbf{2 a}$, and $(R)$-2d were prepared. Excellent yields and enantioselectivities were achieved using chiral thiourea-tertiary amines $\mathbf{3 a}$ and $\mathbf{3 b}$ as the catalysts (Scheme 3) [11].

2-Aminopyranopyridine-3-carbonitriles $\mathbf{2 h - 2 n}$ derived from monoenones $\mathbf{1 h}-\mathbf{1 n}$ were also prepared (Scheme 4) [12]. Cyclic enones $\mathbf{1 h}-\mathbf{1 j}$ reacted with malononitrile in the presence of triethylamine. The reaction of acyclic enones 1k-1n was achieved using piperidine as the catalyst. Generally products $\mathbf{2 h}-\mathbf{2 n}$ were obtained in good yields.

For a further understanding the effect of $\mathrm{C}\left(s p^{3}\right)$ chiral structure in 2-aminopyranopyridine-3-carbonitriles, the compounds $\mathbf{4 a}$ and $\mathbf{4 j}$ with achiral pyridine structure were designed. The treatment of 2-amino-pyran $2 \mathbf{a}$ and $\mathbf{2 j}$ with ammonium acetate provided $\mathbf{4 a}$ and $\mathbf{4 j}$ in good yields (Scheme 5) [13].

\subsection{Evaluation of Antitubercular Activity}

The antitubercular activity of racemic 2 -amino- $4 H$-pyran3-carbonitriles 2a-2e, homochiral 2-amino-4H-pyran-3carbonitriles $(S)-\mathbf{2 a},(S)-\mathbf{2 d},(R)-\mathbf{2 a}$, and $(R)-\mathbf{2 d}$ were evaluated against autoluminescent $M$. tuberculosis H37Ra [14]. This screen model is fast and cost-efficient for the preliminary evaluation of antitubercular activity. Isoniazid and rifampicin were used as the positive control and the results are listed in Figure 1. The bacteria growth was conveniently monitored by the bioluminescence intensity. Unexpectedly all compounds including 2a and 2d did not showed obvious antitubercualr activity.

We further examined the inhibitive activity of the compounds against standard strain M. tuberculosis H37Rv and the results are summarized in Table 1. Perumal and co-workers reported that compound $\mathbf{2 a}$ and $\mathbf{2 d}$ possess excellent antitubercular activities (MIC 0.97 and 0.92 $\mu \mathrm{g} / \mathrm{mL}$ against $\mathrm{H} 37 \mathrm{Rv}$ respectively) [9]. Our present study led to significantly different results. Racemic 2a, 2d and their homochiral enantiomers did not show obvious antitubercular activities (MIC $>10 \mu \mathrm{g} / \mathrm{mL}$ ). Other structural analogs $\mathbf{2 b}, \mathbf{2 c}, \mathbf{2 e - 2 g}$ also appeared to be inefficient. The compounds $\mathbf{2 h}-\mathbf{2 n}$ and $\mathbf{4 a}, \mathbf{4 j}$ with further structural diversities still showed disappointed antitubercular activities. These results brought the question about the reported antitubercular activity of 2-amino-4H-pyran3-carbonitriles by Perumal and co-workers. Although a clear conclusion could not be achieved so far, the further examination of the reported data is highly desirable.

\section{Conclusion}

In conclusion, we designed and synthesized a series of 2amino-4H-pyran-3-carbonitriles and their structural analogs. Homochiral 2-amino-4H-pyran-3-carbonitriles were also prepared via the organocatalytic enantioselctive reaction. The antitubercualr activities of these compounds were determined against autoluminescent $M$. tuberculosis H37Ra and standard strain M. tuberculosis H37Rv, however, no obvious inhibitive activities could be observed. The results are in sharp contrast with the previously reported data. Before the further attempt to develop 2amino-4H-pyran-3-carbonitriles as potential antitubercular agents, the clarification of the contradictive activity data is required.

\section{Experimentals}

${ }^{1} \mathrm{H}$ and ${ }^{13} \mathrm{C}$ NMR spectra were recorded on a Bruker Advance $400 \mathrm{MHz}$ spectrometer as solutions in $\mathrm{CDCl}_{3}$. Chemical shifts in ${ }^{1} \mathrm{H}$ NMR spectra are reported in parts per million (ppm, $\delta$ ) downfield from the internal standard $\mathrm{Me}_{4} \mathrm{Si}$ (TMS, $\delta=0 \mathrm{ppm}$ ). Chemical shifts in ${ }^{13} \mathrm{C} \mathrm{NMR}$ spectra are reported relative to the central line of the chloroform signal ( $\delta=77.0 \mathrm{ppm})$. The following abbreviations are used to designate chemical shift mutiplicities: $\mathrm{s}=$ singlet, $\mathrm{d}=$ doublet, $\mathrm{m}=$ multiplet. High-resolution mass spectra were obtained with Shimadazu LCMS-ITTOF mass spectrometer. Infrared (IR) spectra were recorded on a Bruker Tensor 37 spectrophotometer. Data are represented as follows: frequency of absorption $\left(\mathrm{cm}^{-1}\right)$, intensity of absorption $(\mathrm{s}=$ strong, $\mathrm{m}=$ medium, $\mathrm{w}=$ weak). The flash column chromatography was car- 


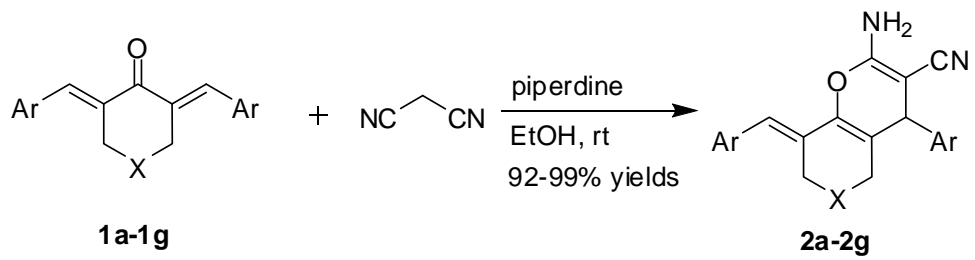

2a: $\mathrm{Ar}=4-\mathrm{F}-\mathrm{C}_{6} \mathrm{H}_{4}, \mathrm{X}=\mathrm{CH}_{3} \mathrm{~N} ; \mathbf{2} \mathbf{b}: \mathrm{Ar}=3-\mathrm{F}-\mathrm{C}_{6} \mathrm{H}_{4}, \mathrm{X}=\mathrm{CH}_{3} \mathrm{~N}$;

2c: $\mathrm{Ar}=3,4-\mathrm{diF}-\mathrm{C}_{6} \mathrm{H}_{3}, \mathrm{X}=\mathrm{CH}_{3} \mathrm{~N} ; \mathbf{2 d}$ : $\mathrm{Ar}=4-\mathrm{Cl}-\mathrm{C}_{6} \mathrm{H}_{4}, \mathrm{X}=\mathrm{CH}_{3} \mathrm{~N}$;

2e: $\mathrm{Ar}=4-\mathrm{F}-\mathrm{C}_{6} \mathrm{H}_{4}, \mathrm{X}=\mathrm{O} ; \mathbf{2 f}: \mathrm{Ar}=4-\mathrm{F}-\mathrm{C}_{6} \mathrm{H}_{4}, \mathrm{X}=\mathrm{S} ; \mathbf{2 g}: \mathrm{Ar}=4-\mathrm{F}-\mathrm{C}_{6} \mathrm{H}_{4}, \mathrm{X}=\mathrm{CH}_{2}$

Scheme 2. Synthesis of 2-amino-4H-pyran-3-carbonitriles 2a-2g.<smiles>CN1CC(=CBr)C(=O)C(=C[Bi])CN1C</smiles>

$1 a, 1 d$

$(R)-2 \mathrm{a}, \mathbf{2 d}$ and $(S)-2 \mathrm{a}, \mathbf{2 d}$<smiles>FC(F)(F)c1cc(NC(=S)N[C@H]2CCCC[C@H]2N2CCCCC2)cc(C(F)(F)F)c1</smiles><smiles>FC(F)(F)c1cc(NC(=S)N[C@H]2CCCC[C@H]2N2CCCCC2)cc(C(F)(F)F)c1</smiles>

Scheme 3. Synthesis of homochiral 2-amino-4H-pyran-3-carbonitriles.

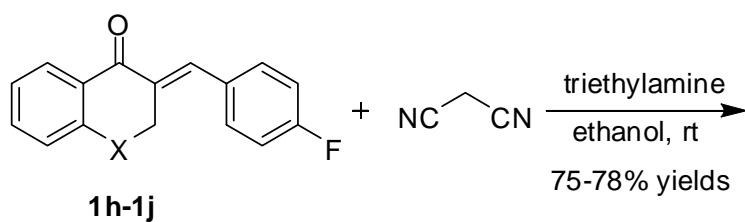

2h: $X=\mathrm{CH}_{2} ; 2 \mathbf{i}: \mathrm{X}=\mathrm{O} ; \mathbf{2 j}: \mathrm{X}=\mathrm{S}$<smiles>[X]c1ccccc1C1=C(CC)C(c2ccc(F)cc2)C(C#N)=C(N)O1</smiles>

2h-2j<smiles>[R]C=C([R])C([R])=O</smiles>

1k-1n<smiles>[R]C1=C([R])C([R])C(C#N)=C(N)O1</smiles>

$2 k-2 n$

2k: $\mathrm{R}^{1}=\mathrm{Ph}, \mathrm{R}^{2}=\mathrm{CN}, \mathrm{R}^{3}=4-\mathrm{F}-\mathrm{C}_{6} \mathrm{H}_{4} ; \mathbf{2 l}: \mathrm{R}^{1}=\mathrm{Me}, \mathrm{R}^{2}=\mathrm{Ph}, \mathrm{R}^{3}=\mathrm{Ph}$;

2m: $\mathrm{R}^{1}=\mathrm{Ph}, \mathrm{R}^{2}=\mathrm{Ph}, \mathrm{R}^{3}=4-\mathrm{Cl}-\mathrm{C}_{6} \mathrm{H}_{4} ; 2 \mathrm{n}: \mathrm{R}^{1}=\mathrm{Ph}, \mathrm{R}^{2}=\mathrm{Ph}, \mathrm{R}^{3}=2$-thiophenyl

Scheme 4. Synthesis of 2-amino- $4 \mathrm{H}$-pyran-3-carbonitriles $2 \mathrm{~h}$-2n derived from monoenones.

ried out over silica gel (230 - 400 mesh), purchased from Qingdao Haiyang Chemical Co. Ltd. Melting points were recorded on an electrothermal digital melting point apparatus and were uncorrected. TLC analysis was performed on precoated silica gel GF254 slides, and visualised by either UV irradiation. Unless otherwise stated, all reagents were obtained from commercial sources and used as received. The solvents were used as commercial anhydrous grade without further purification. Enantiomeric excesses were determined by HPLC using a Daicel Chiralpak AD-H column $(4.6 \mathrm{~mm} \times 25 \mathrm{~cm})$ and eluting with hexane/2-PrOH solution. 


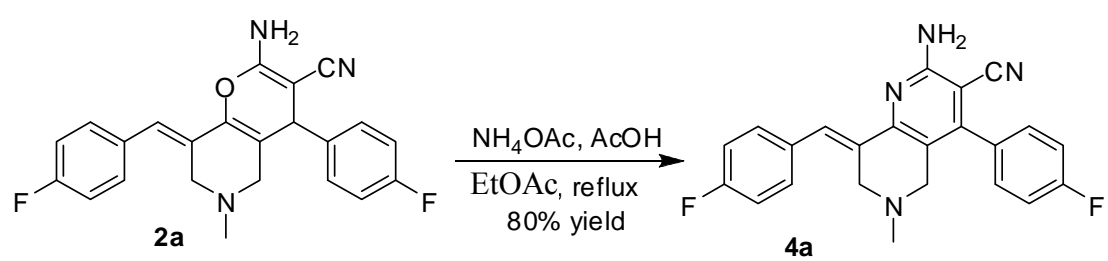<smiles>N#CC1=C(N)OC2=C(CSc3ccccc32)C1c1ccc(F)cc1</smiles>

2j<smiles>CCOC(=O)C(C)CCCCCCC(=O)OO</smiles>

4j

Scheme 5 . Synthesis of pyridine analogs $4 a$ and $4 j$.

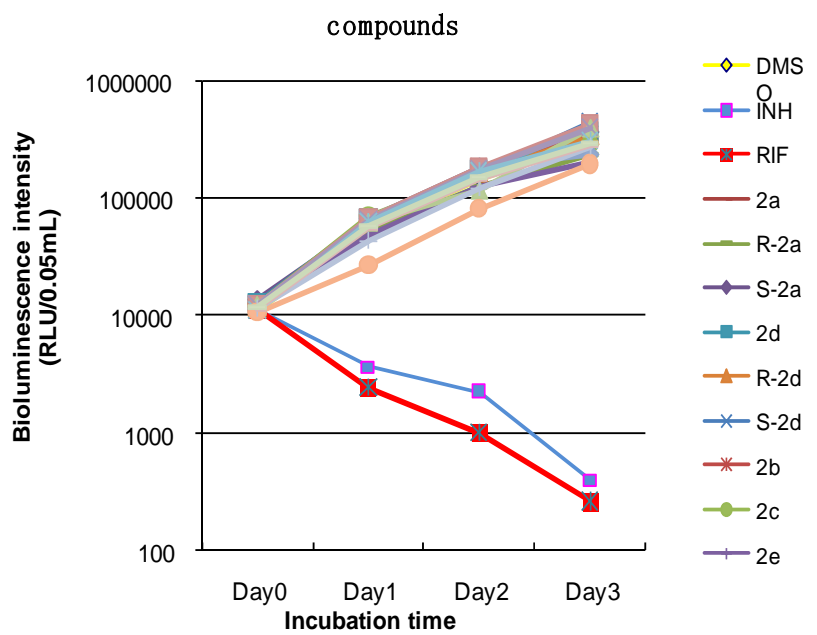

Figure 1. Inhibitive activity against autoluminescent $M$. tuberculosis H37Ra, (INH = isoniazid, RIF = rifampicin. All the compounds were tested at the concentration of $10 \mu \mathrm{g} /$ mL).

\subsection{Typical Procedure for the Synthesis of Compounds 2a-2g}

A mixture of ( $3 E, 5 E)$-3,5-bis(4-fluorobenzylidene)-1methylpiperidin-4-one 1a $(66.1 \mathrm{mg}, 0.2 \mathrm{mmol})$, malononitrile $(19.8 \mathrm{mg}, 0.3 \mathrm{mmol})$ and piperidine $(17.0 \mathrm{mg}, 0.2$ $\mathrm{mmol})$ in ethanol $(2 \mathrm{~mL})$ were stirred for $12 \mathrm{~h}$ at room temperature. The precipitate was filtered to provide $2 \mathbf{a}$ as a white solid.

\subsection{1. (E)-2-Amino-8-(4-Fluorobenzylidene)-4- \\ (4-Fluorophenyl)-6-Methyl-5,6,7,8- \\ Tetrahydro-4H-Pyrano-[3,2-c]Pyridine-3- \\ Carbonitrile (2a) [11]}

White solid, yield $94 \%, \mathrm{mp} 197^{\circ} \mathrm{C}-198^{\circ} \mathrm{C} ;{ }^{1} \mathrm{H}$ NMR $\left(400 \mathrm{MHz}, \mathrm{CDCl}_{3}\right): \delta=7.24-7.17$ (4H, m, ArH), 7.08$7.02(4 \mathrm{H}, \mathrm{m}, \mathrm{ArH}), 6.86(1 \mathrm{H}, \mathrm{s}, \mathrm{HC}=\mathrm{C}), 4.55(2 \mathrm{H}, \mathrm{s}$, $\left.\mathrm{NH}_{2}\right), 4.03(1 \mathrm{H}, \mathrm{s}, \mathrm{CH}), 3.52\left(1 \mathrm{H}, \mathrm{d}, J=13.4 \mathrm{~Hz}, \mathrm{CH}_{2}\right)$, $3.36\left(1 \mathrm{H}, \mathrm{d}, J=13.0 \mathrm{~Hz}, \mathrm{CH}_{2}\right), 2.95(1 \mathrm{H}, \mathrm{d}, J=15.9 \mathrm{~Hz}$,
$\left.\mathrm{CH}_{2}\right), 2.72\left(1 \mathrm{H}, \mathrm{d}, J=15.4 \mathrm{~Hz}, \mathrm{CH}_{2}\right), 2.28\left(3 \mathrm{H}, \mathrm{s}, \mathrm{CH}_{3}\right)$.

\subsection{2. (E)-2-Amino-8-(3-Fluorobenzylidene)-4- (3-Fluorophenyl)-6-Methyl-5,6,7,8- Tetrahydro-4H-Pyrano-[3,2-c]Pyridine-3- Carbonitrile (2b) [9]}

White solid, yield $92 \%, \mathrm{mp} 169^{\circ} \mathrm{C}-172^{\circ} \mathrm{C}$; ${ }^{1} \mathrm{H}$ NMR $\left(400 \mathrm{MHz}, \mathrm{CDCl}_{3}\right): \delta \mathrm{CDCl}_{3}=7.33(2 \mathrm{H}, \mathrm{d}, J=5.9 \mathrm{~Hz}$, ArH), $7.08-7.04$ (1H, d, $J=7.6 \mathrm{~Hz}, \mathrm{ArH}), 7.01-6.96$ $(4 \mathrm{H}, \mathrm{t}, J=19.2 \mathrm{~Hz}, \mathrm{ArH}), 6.93-6.91(1 \mathrm{H}, \mathrm{d}, J=9.6 \mathrm{~Hz}$, ArH), $6.85(1 \mathrm{H}, \mathrm{s}, \mathrm{HC}=\mathrm{C}), 4.59\left(2 \mathrm{H}, \mathrm{s}, \mathrm{NH}_{2}\right), 4.04(1 \mathrm{H}, \mathrm{s}$, $\mathrm{CH}), 3.55\left(1 \mathrm{H}, \mathrm{d}, J=14.4 \mathrm{~Hz}, \mathrm{CH}_{2}\right), 3.36(1 \mathrm{H}, \mathrm{d}, J=$ $\left.14.6 \mathrm{~Hz}, \mathrm{CH}_{2}\right), 2.97\left(1 \mathrm{H}, \mathrm{d}, J=15.8 \mathrm{~Hz}, \mathrm{CH}_{2}\right), 2.74(1 \mathrm{H}$, $\left.\mathrm{d}, J=16.0 \mathrm{~Hz}, \mathrm{CH}_{2}\right), 2.29\left(3 \mathrm{H}, \mathrm{s}, \mathrm{CH}_{3}\right)$.

\subsection{3. (E)-2-Amino-8-(3,4-Difluorobenzylidene)-4- (3,4-Difluorophenyl)-6-methyl-5,6,7,8- \\ Tetrahydro-4H-Pyrano[3,2-c]Pyridine-3- Carbonitrile (2c)}

White solid, yield $92 \%$, mp $207^{\circ} \mathrm{C}-209^{\circ} \mathrm{C}$; ${ }^{1} \mathrm{H}$ NMR (400 MHz, CDCl3): $\delta=7.19-6.94(6 \mathrm{H}, \mathrm{m}, \mathrm{ArH}), 6.80$ $(1 \mathrm{H}, \mathrm{s}, \mathrm{HC}=\mathrm{C}), 4.63\left(2 \mathrm{H}, \mathrm{s}, \mathrm{NH}_{2}\right), 4.01(1 \mathrm{H}, \mathrm{s}, \mathrm{CH}), 3.50$ $\left(1 \mathrm{H}, \mathrm{d}, J=13.8 \mathrm{~Hz}, \mathrm{CH}_{2}\right), 3.34\left(1 \mathrm{H}, \mathrm{d}, J=14.0 \mathrm{~Hz}, \mathrm{CH}_{2}\right)$, $2.96\left(1 \mathrm{H}, \mathrm{d}, J=16.1 \mathrm{~Hz}, \mathrm{CH}_{2}\right), 2.72(1 \mathrm{H}, \mathrm{d}, J=15.8 \mathrm{~Hz}$, $\left.\mathrm{CH}_{2}\right), 2.30\left(3 \mathrm{H}, \mathrm{s}, \mathrm{CH}_{3}\right) ;{ }^{13} \mathrm{C} \mathrm{NMR}\left(100 \mathrm{MHz}, \mathrm{CDCl}_{3}\right): \delta$ $=158.77,140.11,139.09,133.02,127.70,125.36,123.78$, $121.27,119.07,117.92,117.75,117.74,117.60,117.42$ 117.25, 116.72, 112.66, 60.09, 55.13, 54.47, 44.90, 41.09; IR (KBr) $v / \mathrm{cm}^{-1}: 3484(\mathrm{~m}), 2194(\mathrm{~s}), 1681(\mathrm{~m}), 1644(\mathrm{~m})$, 1595 (m), 1517 (s), 1396 (w), 1264 (w), 1110 (m), 910 (w), 785 (w); HRMS (ESI) calcd for $\mathrm{C}_{23} \mathrm{H}_{17} \mathrm{~N}_{3} \mathrm{OF}_{4}^{+}[\mathrm{M}+$ $\mathrm{H}]^{+}$: 428.1381, found: 428.1389 .

\subsection{4. (E)-2-Amino-8-(4-Chlorobenzylidene)-4- (4-Chlorophe-nyl)-6-Methyl-5,6,7,8- Tetrahydro-4H-Pyrano[3,2-c]Pyridine-3- Carbonitrile (2d) [11]}

White solid, yield $96 \%, \mathrm{mp} 205^{\circ} \mathrm{C}-207^{\circ} \mathrm{C}$; ${ }^{1} \mathrm{H}$ NMR (400 MHz, CDCl3): $\delta=7.35$ - 7.32 (4H, m, ArH), 7.21 - 
Table 1. Evaluation of antitubercular activity against $M$. tuberculosis H37Rv.

\begin{tabular}{|c|c|}
\hline Compounds & $\operatorname{MIC}(\mu \mathrm{g} / \mathrm{mL})$ \\
\hline $2 a$ & $>10$ \\
\hline $2 \mathbf{b}$ & $>10$ \\
\hline $2 c$ & $>10$ \\
\hline 2d & $>10$ \\
\hline $2 e$ & $>10$ \\
\hline $2 f$ & $>10$ \\
\hline $2 g$ & $>10$ \\
\hline$(R)-2 a$ & $>10$ \\
\hline$(R)-2 d$ & $>10$ \\
\hline$(S)-2 a$ & $>10$ \\
\hline$(S)-2 d$ & $>10$ \\
\hline $2 h$ & $>10$ \\
\hline $2 \mathbf{i}$ & $>10$ \\
\hline $2 \mathbf{j}$ & $>10$ \\
\hline $2 k$ & $>10$ \\
\hline 21 & $>10$ \\
\hline $2 m$ & $>10$ \\
\hline $2 n$ & $>10$ \\
\hline $4 a$ & $>10$ \\
\hline $4 j$ & $>10$ \\
\hline Isoniazid & 0.03 \\
\hline Rifampicin & $<0.25$ \\
\hline
\end{tabular}

$7.13(4 \mathrm{H}, \mathrm{m}, \mathrm{ArH}), 6.84(1 \mathrm{H}, \mathrm{s}, \mathrm{HC}=\mathrm{C}), 4.57(2 \mathrm{H}, \mathrm{s}$, $\left.\mathrm{NH}_{2}\right), 4.02(1 \mathrm{H}, \mathrm{s}, \mathrm{CH}), 3.51\left(1 \mathrm{H}, \mathrm{d}, J=13.8 \mathrm{~Hz}, \mathrm{CH}_{2}\right)$, $3.35\left(1 \mathrm{H}, \mathrm{d}, J=13.6 \mathrm{~Hz}, \mathrm{CH}_{2}\right), 2.94(1 \mathrm{H}, \mathrm{d}, J=16.0 \mathrm{~Hz}$, $\left.\mathrm{CH}_{2}\right), 2.72\left(1 \mathrm{H}, \mathrm{d}, J=16.0 \mathrm{~Hz}, \mathrm{CH}_{2}\right), 2.27\left(3 \mathrm{H}, \mathrm{s}, \mathrm{CH}_{3}\right)$.

\subsection{5. (E)2-Amino-8-(4-Fluorobenzylidene)-4- (4-Fluorophenyl)-4,5,7,8-Tetrahydropyrano [4,3-b]Pyran-3-Carbonitrile (2e)}

While solid, yield $99 \%, \mathrm{mp} 221^{\circ} \mathrm{C}-223^{\circ} \mathrm{C} ;{ }^{1} \mathrm{H}$ NMR (400 MHz, DMSO-d6): $\delta=7.44$ - 7.05 (8H, m, ArH), $6.92(1 \mathrm{H}, \mathrm{s}, \mathrm{HC}=\mathrm{C}), 6.90\left(2 \mathrm{H}, \mathrm{s}, \mathrm{NH}_{2}\right), 4.59(1 \mathrm{H}, \mathrm{d}, J=$ $\left.14.0 \mathrm{~Hz}, \mathrm{CH}_{2}\right), 4.48\left(1 \mathrm{H}, \mathrm{d}, J=13.9 \mathrm{~Hz}, \mathrm{CH}_{2}\right), 4.17(1 \mathrm{H}$, $\left.\mathrm{d}, J=15.5, \mathrm{CH}_{2}\right), 4.15(1 \mathrm{H}, \mathrm{s}, \mathrm{CH}), 3.72(1 \mathrm{H}, \mathrm{d}, J=15.6$ $\left.\mathrm{Hz}, \mathrm{CH}_{2}\right) ;{ }^{13} \mathrm{C}$ NMR (100 MHz, DMSO): $\delta=162.50$, $160.08,159.70,139.17,138.07,131.72,131.02,130.99$, $129.42,129.30,126.09,120.38,120.15,115.61,115.55$, $115.40,115.34,112.96,65.12,64.90,55.77$; IR (KBr) $v / \mathrm{cm}^{-1}: 3472(\mathrm{~m}), 3312(\mathrm{w}), 2220$ (s), 1685 (m), 1660 (m), 1603 (s), 1508 (s), 1414 (w), 1391 (w), 1264 (w),
1228 (s), 1155 (w), 1098 (m), 883 (w), 838 (w), $780(\mathrm{w})$, $744(\mathrm{~m})$; HRMS (ESI) calcd for $\mathrm{C}_{22} \mathrm{H}_{16} \mathrm{~N}_{2} \mathrm{O}_{2} \mathrm{~F}_{2}{ }^{+}[\mathrm{M}+$ $\mathrm{Na}]^{+}:$401.1072, found: 401.1065 .

\subsubsection{2-Amino-8-(4-Fluorobenzylidene)-4- (4-Fluorophenyl)-4,5,7,8-Tetrahydrothiopyrano [4,3-b]Pyran-3-Carbonitrile (2f)}

White solid, yield $98 \%, \mathrm{mp} 205^{\circ} \mathrm{C}-207^{\circ} \mathrm{C}$; ${ }^{1} \mathrm{H}$ NMR $\left(400 \mathrm{MHz}, \mathrm{CDCl}_{3}\right): \delta=7.25-7.21(4 \mathrm{H}, \mathrm{m}, \mathrm{ArH}), 7.10-$ $7.03(4 \mathrm{H}, \mathrm{m}, \mathrm{ArH}), 6.93(1 \mathrm{H}, \mathrm{s}, \mathrm{HC}=\mathrm{C}), 4.53(2 \mathrm{H}, \mathrm{s}$, $\left.\mathrm{NH}_{2}\right), 4.03(1 \mathrm{H}, \mathrm{s}, \mathrm{CH}), 3.58\left(2 \mathrm{H}, \mathrm{q}, J=9.0 \mathrm{~Hz}, \mathrm{CH}_{2}\right)$, $3.04\left(2 \mathrm{H}, \mathrm{q}, J=12.0 \mathrm{~Hz}, \mathrm{CH}_{2}\right) ;{ }^{13} \mathrm{C} \mathrm{NMR}(100 \mathrm{MHz}$, DMSO-d6): $\delta=159.65,143.42,141.39,135.84,129.13$, $128.74,128.51,127.51,127.39,127.16,126.35,124.39$, $120.23,114.04,55.97,43.21,27.20,27.15$; IR (KBr) $v / \mathrm{cm}^{-1}: 3452(\mathrm{~m}), 3355(\mathrm{~m}), 2360$ (s), 2192 (m), 2024 (w), 1673 (s), 1627 (w), 1599 (w), 1506 (s), 1410 (m), $1233(\mathrm{~m}), 1126(\mathrm{~m}), 881(\mathrm{w}), 850$ (w); HRMS (ESI) calcd for $\mathrm{C}_{22} \mathrm{H}_{16} \mathrm{~N}_{2} \mathrm{OF}_{2} \mathrm{~S}^{+}[\mathrm{M}+\mathrm{Na}]^{+}$: 417.0844, found: 417.0843 .

\subsection{7. (E)-2-Amino-8-(4-Fluorobenzylidene)-4- (4-Fluorophenyl)-5,6,7,8-Tetrahydro-4H- Chromene-3-Carbonitrile (2g) [10]}

White solid, yield $99 \%, \mathrm{mp} 211^{\circ} \mathrm{C}-213^{\circ} \mathrm{C}:{ }^{1} \mathrm{H} \mathrm{NMR}$ $\left(400 \mathrm{MHz}, \mathrm{CDCl}_{3}\right): \delta=7.25-7.20(4 \mathrm{H}, \mathrm{m}, \mathrm{ArH}), 7.06-$ $7.02(4 \mathrm{H}, \mathrm{m}, \mathrm{ArH}), 6.83(1 \mathrm{H}, \mathrm{s}, \mathrm{HC}=\mathrm{C}), 4.56(2 \mathrm{H}, \mathrm{s}$, $\left.\mathrm{NH}_{2}\right), 3.97(1 \mathrm{H}, \mathrm{s}, \mathrm{CH}), 2.74-2.70\left(1 \mathrm{H}, \mathrm{m}, \mathrm{CH}_{2}\right), 2.60-$ $2.56\left(1 \mathrm{H}, \mathrm{m}, \mathrm{CH}_{2}\right), 2.04-1.92\left(2 \mathrm{H}, \mathrm{m}, \mathrm{CH}_{2}\right), 1.74-1.52$ $\left(2 \mathrm{H}, \mathrm{m}, \mathrm{CH}_{2}\right)$; HRMS (ESI) calcd for $\mathrm{C}_{23} \mathrm{H}_{18} \mathrm{~N}_{2} \mathrm{OF}_{2}{ }^{+}[\mathrm{M}$ $+\mathrm{Na}]^{+}: 399.1279$, found: 399.1261 .

\subsection{Typical Procedure for the Synthesis of Homochiral Compounds (S)-2a, (S)-2d, (R)-2a, and (R)-2d}

A mixture of $(3 E, 5 E)-3,5$-bis(4-fluorobenzylidene)-1methylpiperidin-4-one 1a (66.1 mg, $0.2 \mathrm{mmol})$, malononitrile $(19.8 \mathrm{mg}, 0.3 \mathrm{mmol})$ and $3 \mathbf{a}(9.0 \mathrm{mg}, 0.02 \mathrm{mmol})$ in toluene $(2 \mathrm{~mL})$ were stirred for $28 \mathrm{~h}$ at room temperature. The white precipitate $(S)$-2a was collected by the centrifugalization.

\subsection{1. (S,E)-2-Amino-8-(4-Fluorobenzylidene)-4- (4-Fluorophenyl)-6-Methyl-5,6,7,8-Tetrahydro- 4H-Pyrano-[3,2-c]Pyridine-3-Carbonitrile (S-2a) [11]}

White solid, yield $94 \%, \mathrm{mp} 197^{\circ} \mathrm{C}-198^{\circ} \mathrm{C}$; ${ }^{1} \mathrm{H}$ NMR $\left(400 \mathrm{MHz}, \mathrm{CDCl}_{3}\right): \delta=7.24-7.17(4 \mathrm{H}, \mathrm{m}, \mathrm{ArH}), 7.08-$ $7.02(4 \mathrm{H}, \mathrm{m}, \mathrm{ArH}), 6.86(1 \mathrm{H}, \mathrm{s}, \mathrm{HC}=\mathrm{C}), 4.55(2 \mathrm{H}, \mathrm{s}$, $\left.\mathrm{NH}_{2}\right), 4.03(1 \mathrm{H}, \mathrm{s}, \mathrm{CH}), 3.52\left(1 \mathrm{H}, \mathrm{d}, J=13.4 \mathrm{~Hz}, \mathrm{CH}_{2}\right)$, $3.36\left(1 \mathrm{H}, \mathrm{d}, J=13.0 \mathrm{~Hz}, \mathrm{CH}_{2}\right), 2.95(1 \mathrm{H}, \mathrm{d}, J=15.9 \mathrm{~Hz}$, $\left.\mathrm{CH}_{2}\right), 2.72\left(1 \mathrm{H}, \mathrm{d}, J=15.4 \mathrm{~Hz}, \mathrm{CH}_{2}\right), 2.28\left(3 \mathrm{H}, \mathrm{s}, \mathrm{CH}_{3}\right)$. Enantiomeric excess was determined by HPLC with a CHIRALPAK AD-H column (i-PrOH/hexane = 30:70, 
$254 \mathrm{~nm}, 0.8 \mathrm{~mL} / \mathrm{min}), \mathrm{t}_{\mathrm{r}}($ major $)=10.6 \mathrm{~min}, \mathrm{t}_{\mathrm{r}}($ minor $)=$ $7.5 \mathrm{~min}, 99 \%$ ee.

\subsection{2. (R,E)-2-Amino-8-(4-Fluorobenzylidene)-4- (4-Fluorophenyl)-6-Methyl-5,6,7,8-Tetrahydro- 4H-Pyrano-[3,2-c]Pyridine-3-Carbonitrile (R-2a) [11]}

White solid, yield $94 \%, \mathrm{mp} 197^{\circ} \mathrm{C}-198^{\circ} \mathrm{C}$; ${ }^{1} \mathrm{H}$ NMR $\left(400 \mathrm{MHz}, \mathrm{CDCl}_{3}\right): \delta=7.24-7.17(4 \mathrm{H}, \mathrm{m}, \mathrm{ArH}), 7.08-$ $7.02(4 \mathrm{H}, \mathrm{m}, \mathrm{ArH}), 6.86(1 \mathrm{H}, \mathrm{s}, \mathrm{HC}=\mathrm{C}), 4.55(2 \mathrm{H}, \mathrm{s}$, $\left.\mathrm{NH}_{2}\right), 4.03(1 \mathrm{H}, \mathrm{s}, \mathrm{CH}), 3.52\left(1 \mathrm{H}, \mathrm{d}, J=13.4 \mathrm{~Hz}, \mathrm{CH}_{2}\right)$, $3.36\left(1 \mathrm{H}, \mathrm{d}, J=13.0 \mathrm{~Hz}, \mathrm{CH}_{2}\right), 2.95(1 \mathrm{H}, \mathrm{d}, J=15.9 \mathrm{~Hz}$, $\left.\mathrm{CH}_{2}\right), 2.72\left(1 \mathrm{H}, \mathrm{d}, J=15.4 \mathrm{~Hz}, \mathrm{CH}_{2}\right), 2.28\left(3 \mathrm{H}, \mathrm{s}, \mathrm{CH}_{3}\right)$. Enantiomeric excess was determined by HPLC with a CHIRALPAK AD-H column (i-PrOH/hexane $=30: 70$, $254 \mathrm{~nm}, 0.8 \mathrm{~mL} / \mathrm{min}), \mathrm{t}_{\mathrm{r}}($ major $)=7.5 \mathrm{~min}, \mathrm{t}_{\mathrm{r}}($ minor $)=$ $10.6 \mathrm{~min} .98 \%$ ee.

\subsection{3. (S,E)-2-Amino-8-(4-Chlorobenzylidene)-4- (4-Chlorophe-nyl)-6-Methyl-5,6,7,8- Tetrahydro-4H-Pyrano[3,2-c]Pyridine-3- Carbonitrile (S-2d) [11]}

White solid, yield $96 \%, \mathrm{mp} 205^{\circ} \mathrm{C}-207^{\circ} \mathrm{C} ;{ }^{1} \mathrm{H}$ NMR $\left(400 \mathrm{MHz}, \mathrm{CDCl}_{3}\right): \delta=7.35-7.32$ (4H, m, ArH), 7.21$7.13(4 \mathrm{H}, \mathrm{m}, \mathrm{ArH}), 6.84(1 \mathrm{H}, \mathrm{s}, \mathrm{HC}=\mathrm{C}), 4.57(2 \mathrm{H}, \mathrm{s}$, $\left.\mathrm{NH}_{2}\right), 4.02(1 \mathrm{H}, \mathrm{s}, \mathrm{CH}), 3.51\left(1 \mathrm{H}, \mathrm{d}, J=13.8 \mathrm{~Hz}, \mathrm{CH}_{2}\right)$, $3.35\left(1 \mathrm{H}, \mathrm{d}, J=13.6 \mathrm{~Hz}, \mathrm{CH}_{2}\right), 2.94(1 \mathrm{H}, \mathrm{d}, J=16.0 \mathrm{~Hz}$, $\left.\mathrm{CH}_{2}\right), 2.72\left(1 \mathrm{H}, \mathrm{d}, J=16.0 \mathrm{~Hz}, \mathrm{CH}_{2}\right), 2.27\left(3 \mathrm{H}, \mathrm{s}, \mathrm{CH}_{3}\right)$. Enantiomeric excess was determined by HPLC with a CHIRALPAK AD-H column (i-PrOH/hexane $=30: 70$, $254 \mathrm{~nm}, 0.8 \mathrm{~mL} / \mathrm{min}), \mathrm{t}_{\mathrm{r}}($ major $)=9.7 \mathrm{~min}, \mathrm{t}_{\mathrm{r}}($ minor $)=$ $7.6 \mathrm{~min}, 98 \%$ ee.

\subsection{4. (R,E)-2-Amino-8-(4-Chlorobenzylidene)-4- (4-Chlorophe-nyl)-6-Methyl-5,6,7,8- Tetrahydro-4H-Pyr-ano[3,2-c]Pyridine-3- Carbonitrile (R-2d) [11]}

White solid, yield $96 \%, \mathrm{mp} 205^{\circ} \mathrm{C}-207^{\circ} \mathrm{C}$; $1 \mathrm{H}$ NMR (400 MHz, $\mathrm{CDCl} 3): \delta=7.35-7.32(4 \mathrm{H}, \mathrm{m}, \mathrm{ArH}), 7.21$ $7.13(4 \mathrm{H}, \mathrm{m}, \mathrm{ArH}), 6.84(1 \mathrm{H}, \mathrm{s}, \mathrm{HC}=\mathrm{C}), 4.57(2 \mathrm{H}, \mathrm{s}$, $\left.\mathrm{NH}_{2}\right), 4.02(1 \mathrm{H}, \mathrm{s}, \mathrm{CH}), 3.51\left(1 \mathrm{H}, \mathrm{d}, J=13.8 \mathrm{~Hz}, \mathrm{CH}_{2}\right)$, $3.35\left(1 \mathrm{H}, \mathrm{d}, J=13.6 \mathrm{~Hz}, \mathrm{CH}_{2}\right), 2.94(1 \mathrm{H}, \mathrm{d}, J=16.0 \mathrm{~Hz}$, $\left.\mathrm{CH}_{2}\right), 2.72\left(1 \mathrm{H}, \mathrm{d}, J=16.0 \mathrm{~Hz}, \mathrm{CH}_{2}\right), 2.27\left(3 \mathrm{H}, \mathrm{s}, \mathrm{CH}_{3}\right)$. Enantiomeric excess was determined by HPLC with a CHIRALPAK AD-H column (i-PrOH/hexane $=30: 70$, $254 \mathrm{~nm}, 0.8 \mathrm{~mL} / \mathrm{min}), \mathrm{t}_{\mathrm{r}}($ major $)=7.6 \mathrm{~min}, \mathrm{t}_{\mathrm{r}}($ minor $)=$ $9.7 \mathrm{~min}, 99 \%$ ee.

\subsection{Typical Procedure for the Synthesis of Compounds $2 \mathrm{~h}-\mathbf{2 j}$}

A mixture of (E)-2-(4-fluorobenzylidene)-3,4-dihy-dronaphthalen-1(2H)-one $\mathbf{1 h}(50.5 \mathrm{mg}, 0.2 \mathrm{mmol})$, malononitrile $(19.8 \mathrm{mg}, 0.3 \mathrm{mmol})$ and triethylamine $(20.2 \mathrm{mg}$,
$0.2 \mathrm{mmol})$ in ethanol $(2 \mathrm{~mL})$ were stirred for $12 \mathrm{~h}$ at room temperature. The precipitate was filtered to provide $\mathbf{2 h}$ as a yellow solid.

\subsubsection{2-Amino-4-(4-Fluorophenyl)-5,6-Dihydro-} 4H-Benzo[h]Chromene-3-Carbonitrile (2h) [12] Yellow solid, yield $94 \%, \mathrm{mp} 184^{\circ} \mathrm{C}-186^{\circ} \mathrm{C} ;{ }^{1} \mathrm{H}$ NMR $\left(400 \mathrm{MHz}, \mathrm{CDCl}_{3}\right): \delta=7.46(1 \mathrm{H}, \mathrm{d}, J=7.2 \mathrm{~Hz}, \mathrm{ArH})$, $7.26-7.19(4 \mathrm{H}, \mathrm{m}, \mathrm{ArH}), 7.11(1 \mathrm{H}, \mathrm{d}, J=6.8 \mathrm{~Hz}, \mathrm{ArH})$, $7.01(2 \mathrm{H}, \mathrm{t}, J=8.2 \mathrm{~Hz}, \mathrm{ArH}), 4.58\left(2 \mathrm{H}, \mathrm{s}, \mathrm{NH}_{2}\right), 4.07$ $(1 \mathrm{H}, \mathrm{s}, \mathrm{CH}), 2.81\left(1 \mathrm{H}, \mathrm{dd}, J=15.9,8.1 \mathrm{~Hz}, \mathrm{CH}_{2}\right), 2.69$ $\left(1 \mathrm{H}, \mathrm{dt}, J=15.7,7.8 \mathrm{~Hz}, \mathrm{CH}_{2}\right), 2.16(1 \mathrm{H}, \mathrm{dt}, J=15.9$, $\left.7.9 \mathrm{~Hz}, \mathrm{CH}_{2}\right), 2.09-1.96\left(1 \mathrm{H}, \mathrm{m}, \mathrm{CH}_{2}\right)$; HRMS (ESI) calcd for $\mathrm{C}_{20} \mathrm{H}_{15} \mathrm{~N}_{2} \mathrm{OF}^{+}[\mathrm{M}+\mathrm{Na}]^{+}$: 341.1061, found: 341.1054 .

\subsubsection{2-Amino-4-(4-Fluorophenyl)-4,5-} Dihydropyrano[3,2-c]Chromene-3-Carbonitrile (2i)

Yellow solid, yield $75 \%$, mp $175^{\circ} \mathrm{C}-177^{\circ} \mathrm{C} ;{ }^{1} \mathrm{H}$ NMR $\left(400 \mathrm{MHz}, \mathrm{CDCl}_{3}\right): \delta=7.34(1 \mathrm{H}, \mathrm{d}, J=7.6 \mathrm{~Hz}, \mathrm{ArH})$, $7.30-7.14(3 \mathrm{H}, \mathrm{m}, \mathrm{ArH}), 7.05(2 \mathrm{H}, \mathrm{dd}, J=12.0,5.0 \mathrm{~Hz}$, ArH), $6.96(1 \mathrm{H}, \mathrm{t}, J=7.5 \mathrm{~Hz}, \mathrm{ArH}), 6.80(1 \mathrm{H}, \mathrm{d}, J=8.1$ $\mathrm{Hz}, \mathrm{ArH}), 4.67\left(2 \mathrm{H}, \mathrm{s}, \mathrm{NH}_{2}\right), 4.62-4.55(1 \mathrm{H}, \mathrm{d}, J=13.6$ $\left.\mathrm{Hz}, \mathrm{CH}_{2}\right), 4.41\left(1 \mathrm{H}, \mathrm{d}, J=13.7 \mathrm{~Hz}, \mathrm{CH}_{2}\right), 4.03(1 \mathrm{H}, \mathrm{s}$, $\mathrm{CH}) ;{ }^{13} \mathrm{C}$ NMR $\left(100 \mathrm{MHz}, \mathrm{CDCl}_{3}\right): \delta=163.61,161.17$, $158.86,154.14,138.16,136.81,130.46,129.55,129.48$, 121.36, 121.13, 116.58, 116.08, 116.00, 115.76, 104.75, 66.37, 60.87, 39.07; IR (KBr) v/ $\mathrm{cm}^{-1}: 3327$ (m), 2195 (m), 1709 (s), $1656(\mathrm{~s}), 1600(\mathrm{~s}), 1506(\mathrm{~m}), 1356(\mathrm{~m})$, $1230(\mathrm{~s}), 1157$ (m), $1101(\mathrm{w}), 1036(\mathrm{w}), 836(\mathrm{~m}), 755$ (w); HRMS (ESI) calcd for $\mathrm{C}_{19} \mathrm{H}_{13} \mathrm{~N}_{2} \mathrm{O}_{2} \mathrm{~F}^{+}[\mathrm{M}+\mathrm{Na}]^{+}$: 343.0853, found: 343.0857 .

\subsubsection{2-Amino-4-(4-Fluorophenyl)-4,5- Dihydrothiochromeno[4,3-b]Pyran-3- Carbonitrile (2j)}

Yellow solid, yield $76 \%$, mp $158^{\circ} \mathrm{C}-160^{\circ} \mathrm{C} ;{ }^{1} \mathrm{H}$ NMR $\left(400 \mathrm{MHz}, \mathrm{CDCl}_{3}\right): \delta=7.53(1 \mathrm{H}, \mathrm{d}, J=16.8 \mathrm{~Hz}, \mathrm{ArH})$, $7.36-7.29$ (2H, m, ArH), 7.28 - 7.14 (3H, m, ArH), 7.04 $(2 \mathrm{H}, \mathrm{dd}, J=12.1,4.9 \mathrm{~Hz}, \mathrm{ArH}), 4.63\left(2 \mathrm{H}, \mathrm{s}, \mathrm{NH}_{2}\right), 4.13$ $(1 \mathrm{H}, \mathrm{s}, \mathrm{CH}), 3.29\left(1 \mathrm{H}, \mathrm{d}, J=15.1 \mathrm{~Hz}, \mathrm{CH}_{2}\right), 3.09(1 \mathrm{H}, \mathrm{d}$, $\left.J=15.1 \mathrm{~Hz}, \mathrm{CH}_{2}\right) ;{ }^{13} \mathrm{C}$ NMR $\left(100 \mathrm{MHz}, \mathrm{CDCl}_{3}\right): \delta=$ $163.63,161.18,158.63,142.14,137.53,132.76,129.77$, $129.70,129.11,127.15,125.58,123.28,119.20,116.03$, $115.81,107.77,60.99,42.39,27.00$; IR (KBr) $v / \mathrm{cm}^{-1}$ : 3475 (w), 2360 (w), 2197 (s), 2024 (w), 1692 (s), 1635 (w), $1598(\mathrm{~s}), 1506(\mathrm{w}), 1407(\mathrm{~s}), 1343(\mathrm{w}), 1258(\mathrm{w})$, 1219 (w), 1121 (s), 848 (w), 728 (w); HRMS (ESI) calcd for $\mathrm{C}_{19} \mathrm{H}_{13} \mathrm{~N}_{2} \mathrm{OFS}^{+}[\mathrm{M}+\mathrm{Na}]^{+}: 359.0625$, found: 359.0631 .

\subsection{Typical Procedure for the Synthesis of Compounds 2k-2n}

A mixture of (E)-2-benzoyl-3-(4-fluorophenyl)acryloni- 
itrile 1k (50.2 mg, $0.2 \mathrm{mmol})$, malononitrile (19.8 mg, $0.3 \mathrm{mmol})$, and piperidine $(17.0 \mathrm{mg}, 0.2 \mathrm{mmol})$ in toluene $(2 \mathrm{~mL})$ was stirred at room temperature for $24 \mathrm{~h}$. After the solvent was evaporated, the residue was purified by flash column chromatography (ethyl acetate/petroleum ether $=1: 5$ ) to give $\mathbf{2} \mathbf{k}$.

\subsubsection{2-Amino-4-(4-Fluorophenyl)-6-Phenyl- 4H-Pyran-3,5-Dicarbonitrile (2k)}

Yellow solid, yield $85 \%, \operatorname{mp~} 61{ }^{\circ} \mathrm{C}-63^{\circ} \mathrm{C} ;{ }^{1} \mathrm{H}$ NMR (400 $\left.\mathrm{MHz}, \mathrm{CDCl}_{3}\right): \delta=7.74(2 \mathrm{H}, \mathrm{dd}, J=7.0,1.5 \mathrm{~Hz}, \mathrm{ArH})$, 7.56 - 7.40 (3H, m, ArH), 7.38 - 7.27 (2H, m, ArH), 7.10 (2H, ddd, $J=8.6,5.0,2.7 \mathrm{~Hz}, \mathrm{ArH}), 4.89\left(2 \mathrm{H}, \mathrm{s}, \mathrm{NH}_{2}\right)$, $4.35(1 \mathrm{H}, \mathrm{s}, \mathrm{CH}) ;{ }^{13} \mathrm{C} \mathrm{NMR}\left(100 \mathrm{MHz}, \mathrm{CDCl}_{3}\right): \delta=$ $163.98,161.48,157.86,157.70,136.49,131.96,129.71$, $129.60,129.51,128.81,127.80,117.76,116.83,116.34$, 116.12, 90.70, 59.91, 40.11; IR (KBr) $v / \mathrm{cm}^{-1}: 3333(\mathrm{w})$, 2198 (w), 1673 (s), 1602 (w), 1508 (m), 1401 (m), 1341 (s), 1263 (w), 1081 (m), 743 (w); HRMS (ESI) calcd for $\mathrm{C}_{19} \mathrm{H}_{12} \mathrm{~N}_{3} \mathrm{OF}^{+}[\mathrm{M}+\mathrm{Na}]^{+}: 340.0857$, found: 340.0891 .

\subsubsection{2-Amino-6-Methyl-4,5-Diphenyl-4H-}

Pyran-3-Carbonitrile (2l) [12]

White solid, yield 78\%, ${ }^{1} \mathrm{H}$ NMR (400 MHz, CDCl3): $\delta$ $=7.23-7.13(6 \mathrm{H}, \mathrm{m}, \mathrm{ArH}), 7.09-7.07(2 \mathrm{H}, \mathrm{m}, \mathrm{ArH})$, 6.90 - $6.87(2 \mathrm{H}, \mathrm{m}, \mathrm{ArH}), 4.49\left(2 \mathrm{H}, \mathrm{s}, \mathrm{NH}_{2}\right), 4.18(1 \mathrm{H}, \mathrm{s}$, $\mathrm{CH}), 1.80\left(3 \mathrm{H}, \mathrm{s}, \mathrm{CH}_{3}\right)$.

\subsubsection{2-Amino-4-(4-Chlorophenyl)-5,6-Diphenyl- 4H-Pyran-3-Carbonitrile (2m) [12]}

White solid, yield $80 \%,{ }^{1} \mathrm{H}$ NMR (400 MHz, CDCl3): $\delta$ $=7.26-7.07(12 \mathrm{H}, \mathrm{m}, \mathrm{ArH}), 6.84-6.82(2 \mathrm{H}, \mathrm{m}, \mathrm{ArH})$, $4.56\left(2 \mathrm{H}, \mathrm{s}, \mathrm{NH}_{2}\right), 4.35(1 \mathrm{H}, \mathrm{s}, \mathrm{CH})$.

\subsubsection{2-Amino-5,6-Diphenyl-4-(Thiophen-2-yl)- 4H-Pyran-3-Carbonitrile (2n) [12]}

White solid, yield $86 \%,{ }^{1} \mathrm{H}$ NMR (400 MHz, $\mathrm{CDCl} 3$ ): $\delta$ $=7.25-7.10(9 \mathrm{H}, \mathrm{m}, \mathrm{ArH}), 6.95-6.93(2 \mathrm{H}, \mathrm{m}, \mathrm{ArH})$, 6.87 - 6.85 (1H, m, ArH), $6.79(1 \mathrm{H}, \mathrm{d}, J=2.8, \mathrm{~Hz}, \mathrm{ArH})$, $4.66(1 \mathrm{H}, \mathrm{s}, \mathrm{CH}), 4.58\left(2 \mathrm{H}, \mathrm{s}, \mathrm{NH}_{2}\right)$.

\subsection{Typical Procedure for the Synthesis of Compounds $4 a$ and $4 j$}

A mixture of compound 2a $(39.1 \mathrm{mg}, 0.1 \mathrm{mmol})$, Ac$\mathrm{ONH}_{4}(92 \mathrm{mg}, 1.2 \mathrm{mmol})$, and $\mathrm{AcOH}(1.0 \mathrm{~mL})$ in EtOAc $(1.0 \mathrm{~mL})$ was refluxed for $24 \mathrm{~h}$. After cooled to room temperature, EtOAc $(10 \mathrm{~mL})$ was added. The mixture was washed with saturated aqueous $\mathrm{NaHCO}_{3}(10 \mathrm{~mL})$ and brine $(5 \mathrm{~mL})$, and dried over anhydrous $\mathrm{Na}_{2} \mathrm{SO}_{4}$. After the solvent was evaporated under vacuum, the residue was purified by flash column chromatography (ethyl acetate/petroleum ether $=2: 5$ ) to give the products $4 a$.

\subsection{1. (E)-2-Amino-8-(4-Fluorobenzylidene)-4- \\ (4-Fluorophenyl)-6-Methyl-5,6,7,8-Tetrahydro- 1,6-Naphthyridine-3-Carbonitrile (4a)}

Yellow solid, yield $80 \%$, mp $208^{\circ} \mathrm{C}-210^{\circ} \mathrm{C} ;{ }^{1} \mathrm{H}$ NMR $\left(400 \mathrm{MHz}, \mathrm{CDCl}_{3}\right): \delta=8.02(1 \mathrm{H}, \mathrm{s}, \mathrm{HC}=\mathrm{C}), 7.31-7.10$ $(8 \mathrm{H}, \mathrm{m}, \mathrm{ArH}), 5.15\left(2 \mathrm{H}, \mathrm{s}, \mathrm{NH}_{2}\right), 3.61\left(2 \mathrm{H}, \mathrm{s}, \mathrm{CH}_{2}\right), 3.27$ $\left(2 \mathrm{H}, \mathrm{s}, \mathrm{CH}_{2}\right), 2.35\left(3 \mathrm{H}, \mathrm{s}, \mathrm{CH}_{3}\right) ;{ }^{13} \mathrm{C} \mathrm{NMR}(100 \mathrm{MHz}$, $\left.\mathrm{CDCl}_{3}\right): \delta=164.50,163.38,162.20,161.18,157.36$, $153.20,152.20,132.66,131.78,131.48,131.40,130.21$, $130.13,129.39,116.55,116.22,116.00,115.58,115.37$, 90.78, 55.84, 55.27, 45.52; IR (KBr) $v / \mathrm{cm}^{-1}: 3427(\mathrm{~s})$, $2215(\mathrm{w}), 2024(\mathrm{w}), 1627(\mathrm{~m}), 1602(\mathrm{~m}), 1558(\mathrm{~s}), 1506$ (s), 1423 (w), 1224 (m), 1160 (w), 1103 (s), 917 (w), 829 (w), 558 (m); HRMS (ESI) calcd for $\mathrm{C}_{23} \mathrm{H}_{18} \mathrm{~N}_{4} \mathrm{~F}_{2}{ }^{+}[\mathrm{M}+$ $\mathrm{H}]^{+}:$389.1572, found: 389.1569 .

\subsubsection{2-Amino-4-(4-Fluorophenyl)-5H-Thiochromeno [4,3-b]Pyridine-3-Carbonitrile (4j)}

Yellow solid, yield $85 \%$, mp $202^{\circ} \mathrm{C}-204^{\circ} \mathrm{C}$; ${ }^{1} \mathrm{H}$ NMR $\left(400 \mathrm{MHz}, \mathrm{CDCl}_{3}\right): \delta=8.32(1 \mathrm{H}, \mathrm{d}, J=6.6 \mathrm{~Hz}, \mathrm{ArH})$, 7.51-7.11 (7H, m, ArH), $5.26\left(2 \mathrm{H}, \mathrm{s}, \mathrm{NH}_{2}\right), 3.65(2 \mathrm{H}, \mathrm{s}$, $\left.\mathrm{CH}_{2}\right) ;{ }^{13} \mathrm{C} \mathrm{NMR}\left(100 \mathrm{MHz}, \mathrm{CDCl}_{3}\right): \delta=164.51,162.03$, $158.00,154.68,151.51,136.62,133.51,131.06,130.57$, $130.53,130.50,128.39,127.84,126.26,116.94,116.39$, 116.17, 90.92, 27.21; IR (KBr) $v / \mathrm{cm}^{-1}: 3446$ (s), 2360 (w), 2213 (w), 2023 (w), 1626 (s), 1559 (m), 1427 (m), 1224 (w), 1074 (s), 843 (w), 767 (w), 546 (m); HRMS (ESI) calcd for $\mathrm{C}_{19} \mathrm{H}_{12} \mathrm{~N}_{3} \mathrm{FS}^{+}[\mathrm{M}+\mathrm{H}]^{+}: 334.0809$, found: 334.0804 .

\subsection{Evaluation of Antitubercular Activity}

Autoluminescent $M$. tuberculosis H37Ra was constructed as previously reported [14] and was inoculated in a 50 $\mathrm{mL}$ centrifuge tube containing $5 \mathrm{~mL} 7 \mathrm{H} 9$ with $0.1 \%$ Tween 80 and $10 \%$ ODAC, then incubated at $37^{\circ} \mathrm{C}$ with shaking. When the culture reached an $\mathrm{OD}_{600} \mathrm{~nm}$ of 0.7 , the culture was diluted. $50 \mu \mathrm{L}$ diluted H37Ra were inoculated in sterile 384 well plate. The RLU of each well should be between 8000 - 12000 and was recorded as the basic luminescence of Day 0 . The test compounds and the positive drugs were added to the 384 well plate in triplicate by the Echo520 with the final concentration 1 or $10 \mu \mathrm{g} / \mathrm{mL}$. The luminescent values were detected for the following three days. The data were analyzed with the Excel compared to the DMSO control to estimate the inhibition activity of the compounds.

The antitubercular activities against M. tuberculosis $\mathrm{H} 37 \mathrm{Rv}$ were determined by standard agar dilution method [15].

\section{Acknowledgements}

Financial supports from National Natural Science Foundation of China (Nos. 20972195, 21172270) and Guang- 
dong Engineering Research Center of Chiral Drugs are gratefully acknowledged.

\section{REFERENCES}

[1] World Health Organization, "Global Tuberculosis Control Report 2013," World Health Organization, Geneva. http://www.who.int/tb/publications/global_report/en/

[2] L. Christian, G. Philippe, U. Mukund, L. Knut, G. Haileyesus and R. Mario, "Global Tuberculosis Control: Lessons Learnt and Future Prospects," Nature Reviews Microbiology, Vol. 10, No. 6, 2012, pp. 407-416. http://dx.doi.org/10.1038/nrmicro2797

[3] World Health Organization, "Multidrug-Resistant Tuberculosis (MDR-TB)," World Health Organization, Geneva. http://www.who.int/tb/challenges/mdr/en/index.html

[4] M. P. Grobusch, "Drug-Resistant and Extensively DrugResistant Tuberculosis in Southern Africa," Current Opinion in Pulmonary Medicine, Vol. 16, No. 3, 2010, pp. 180-185. http://dx.doi.org/10.1097/MCP.0b013e3283378680

[5] J. L. Mainardi, J. E. Hugonnet, L. Gutmann and M. Arthur, "Fighting Resistant Tuberculosis with Old Compounds: The Carbapenem Paradigm," Clinical Microbiology and Infection, Vol. 17, No. 12, 2011, pp. 1755-1756. http://dx.doi.org/10.1111/j.1469-0691.2011.03699.x

[6] A. G. Nikalje and P. Mudassar, "Multidrug-Resistant Mycobacterium Tuberculosis: A Brief Review," Asian Journal of Biological Sciences, Vol. 4, No. 2, 2011, pp. 101115. http://dx.doi.org/10.3923/ajbs.2011.101.115

[7] R. Shi and I. Sugawara, "Development of New AntiTuberculosis Drug Candidates," Tohoku Journal of Experimental Medicine, Vol. 221, No. 2, 2010, pp. 97-106. http://dx.doi.org/10.1620/tjem.221.97

[8] S. T. Cole and G. Riccardi, "New Tuberculosis Drugs on the Horizon," Current Opinion in Microbiology, Vol. 14, No. 5, 2011, pp. 570-576.

[9] R. R. Kumar, S. Perumal, P. Senthilkumar, P. Yogeeswari and D. Sriram, "An Atomefficient, Solvent-Free, Green
Synthesis and Antimycobacterial evaluation of 2-amino-6Methyl-4-Aryl-8-[(E)-Arylmethylidene]-5,6,7,8-Tetrahy-Dro4H-Pyrano[3,2-c]Pyridine-3-Carbonitriles," Bioorganic \& Medicinal Chemistry Letters, Vol. 17, No. 23, 2007, pp. 6459-6462.

http://dx.doi.org/10.1016/j.bmcl.2007.09.095

[10] R. R. Kumar, S. Perumal, J. C. Menendez, P. Yogeeswari and D. Sriram, "Antimycobacterial Activity of Novel 1,2,4Oxadiazole-Pyranopyridine/Chromene Hybrids Generated by Chemoselective 1,3-Dipolar Cycloadditions of Nitrile Oxides," Bioorganic \& Medicinal Chemistry Letters, Vol. 19, No. 11, 2011, pp. 3444-3450. http://dx.doi.org/10.1016/j.bmc.2011.04.033

[11] Z. P. Hu, C. L. Lou, J. J. Wang, C. X. Chen and M. Yan, "Organocatalytic Conjugate Addition of Malononitrile to Conformationally Restricted Dienones," The Journal of Organic Chemistry, Vol. 76, No. 10, 2011, pp. 37973804.

[12] Z. P. Hu, W. J. Wang, X. G. Yin, X. J. Zhang and M. Yan, "Enantioselective Synthesis of 2-Amino-4H-Pyrans via the Organocatalytic Cascade Reaction of Malononitrile and A-Substituted Chalcones," Tetrahedron: Asymmetry, Vol. 23, No. 22-23, 2012, pp. 461-467.

[13] H. F. Wang, P. Li, H. F. Cui, X. W. Wang, J. K. Zhang, W. Liu and G. Zhao, "Highly Enantioselective Synthesis of A-Trichloromethyldihydropyrans Catalyzed by Bifunctional Organocatalysts," Tetrahedron, Vol. 67, No. 10, 2011, pp. 1774-1780. http://dx.doi.org/10.1016/j.tet.2011.01.043

[14] T. Y. Zhang, S. Y. Li and E. L. Nuermberger, "Autoluminescent Mycobacterium Tuberculosis for Rapid, RealTime, Non-Invasive Assessment of Drug and Vaccine Efficacy," PLoS One, Vol. 7, No. 1, 2012, Article ID: e29774. http://dx.doi.org/10.1371/journal.pone.0029774

[15] National Committee for Clinical Laboratory Standards, "Susceptibility Testing of Mycobacteria, Nocardia, and Other Aerobic Actinomycetes; Approved Standard," 2nd Edition, CLSI Document M24-A2, Vol. 31, No. 5, 2011, pp. 3-19. 